Research Article

\title{
Mathematical Model for Charpy Impact Energy of V-Notch Specimens
}

\author{
Wei Wang, Ping Wang, Xuesong Liu, Zhibo Dong, and Hongyuan Fang \\ State Key Laboratory of Advanced Welding and Joining, Harbin Institute of Technology, Harbin 150001, China \\ Correspondence should be addressed to Hongyuan Fang; one4321@126.com
}

Received 30 September 2021; Accepted 15 November 2021; Published 2 December 2021

Academic Editor: Song Jiang

Copyright (c) 2021 Wei Wang et al. This is an open access article distributed under the Creative Commons Attribution License, which permits unrestricted use, distribution, and reproduction in any medium, provided the original work is properly cited.

Firstly, by analyzing the response of Charpy V-notch specimen impacted by pendulum, the relationship between specimen geometry, material properties, and impact energy is established and simplified, and the mathematical model for evaluating impact energy of specimens with different sizes is established. Then, the effectiveness of the model through a series of impact tests is verified. Theoretical analysis and experimental results show that the relationship between ligament length and impact energy is quadratic, while the relationship between ligament thickness and impact energy is linear. In the derivation process, the intrinsic impact toughness is used to evaluate the toughness of materials. The mathematical model makes it possible to evaluate the impact energy of specimens with different sizes and provides a theoretical basis for evaluating the impact resistance of structures.

\section{Introduction}

Impact energy characterizes the ability of a structure to consume energy through deformation and fracture under impact load. The impact energy $A_{\mathrm{k}}$, a value measured by the Charpy impact test, is mainly used to control the quality of metallurgical and thermal processing products and determine the ductile-brittle transition temperature of materials [1]. However, the determination of the $A_{\mathrm{k}}$ value is required to use specimens with a certain standard size. The comparison between the impact energy of specimens of different sizes or nonstandard sizes is disapproved. Since it partly depends on the geometry of specimens, impact energy cannot be regarded as a mechanical property of the material itself. Meanwhile, the relationship between impact energy and specimen size is not clear, which cannot be applied to evaluate the impact bearing capacity of structures whose sizes are not the same as standard specimens.

Several methods have been proposed to isolate the impact of collision energy on geometry. Traditionally, impact toughness $\alpha_{\mathrm{k}}$, the quotient of the impact energy, and the cross-sectional area of the notch are used as the parameters to evaluate the toughness of material. However, for material with plastic deformation, energy consumption does not occur on the notched section only, and energy consumption across the section is not uniform, indicating that the impact toughness is not relevant and accurate enough as a mechanical parameter of material. Mikhail et al. [2] studied the influence of different specimen sizes on the impact test results and presented a procedure for transforming data from subsize specimens to data from full-size specimens. Sokolove et al. [3] studied the correlation between the ductile-brittle transition temperatures measured with impact specimens of different sizes, developed a procedure for transforming data from subsize and the full-size specimens, and turned out a good agreement. Lucon et al. [4] found that there is a linear relation between the impact energy and the specimen size by studying the results of the impact test between the small-size specimen and the full-size specimen. Schill et al. [4] studied the correlation between the impact energy of a certain ferritic steel small-size specimen and a full-size standard impact specimen and evaluated the estimation effects of various conversion formulas. Konopik et al. [5] established the conversion relation between the impact test results of small-size impact specimens and full-size standard impact specimens based on the test results. Yang et al. [6] used GA-NN combined model to predict the Charpy impact energy, and R. Muscat et al. [7] used 
membership function modeling to predict the Charpy impact energy of heat-treated steel more accurately. Chaouadi and Fabry [8] did a preliminary study on the relation between the impact energy and the size of the simply supported rectangular cross-section structure under impact load, and the result of the study is that the impact energy is proportional to the section modulus in bending. Sreenivasan and Mannan [9] studied the impact energy of specimens with different cross-sectional shapes. He further verified that the impact energy is proportional to the section modulus in bending of rectangular cross-section structures and found that the regularity is not significant for nonrectangular cross-sections. Inspired by summarizing the existing research, we try to find the functional relationship between the impact sample size and the impact energy measurement results, so as to estimate the impact energy of any size sample.

In this study, based on the load-bearing form of the $\mathrm{V}$-notch specimens subjected to the pendulum lateral impact, and the response process of structural deformation and failure, a mathematical model containing impact energy, mechanical properties, and geometrical parameters of the structure was established. In this way, the influence of specimen geometry to impact energy is extracted, and a new mechanical property to describe the impact load-bearing capability of a material is defined. Finally, the regression analyses based on impact test results of two kinds of steel were performed to verify the rationality of the model.

\section{Mathematical Model to Evaluate Impact Energy}

2.1. Dynamic Response Process of V-Notched Specimen Subjected to Lateral Impact. The deformation mode of V-notch specimen subjected to lateral impact, as shown in Figure 1, can be regarded as a dynamic 3-point bending. After impacted by the pendulum, the dynamic response process of the specimen mainly includes three parts before completely broken: elastic deformation, plastic deformation, and crack propagation. Based on the plane assumption and the analysis of the stress-strain state of the bending deformation, during the elastic deformation, the kinetic energy of the pendulum is transformed into elastic potential energy, which is distributed in the entire specimen. In the plastic deformation stage, the plastic deformation locally occurs near the section which contains the impact position. The point at the tip of the $\mathrm{V}$-notch has the maximum tensile stress and strain. When the bending deformation develops to a certain extent, cracks should occur at this point. After crack initiation, the crack shall propagate in the direction most conducive to its propagation until the sample is completely broken.

Through the analysis of the dynamic response process of the specimen after the impact, it can be known that the resistance capability of the specimen structure to impact load is related to the bending resistance in the elastic and plastic deformation stage and the energy absorption during the crack propagation.

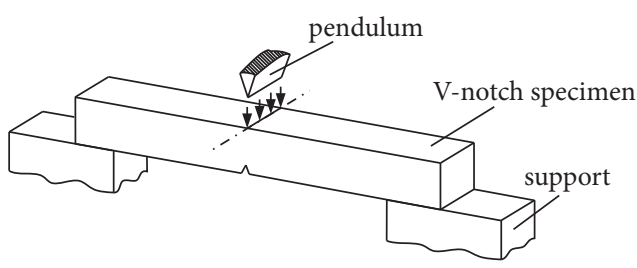

FIgURE 1: V-notch specimen struck by a pendulum transversely.

\subsection{Construction of Mathematical Model of V-Notch Impact}

Energy. Based on the above analysis, it can be concluded that the impact energy consists of three parts: the elastic deformation energy $E_{\mathrm{e}}$, the plastic deformation energy of the structure $E_{\mathrm{p}}$, and the energy consumed by crack propagation in fracture section $E_{\mathrm{c}}$. The impact energy of the specimen can be expressed as the sum of these energies, that is,

$$
A=E_{e}+E_{p}+E_{c} .
$$

In the bending process, energy consumed in the deformation process is the product of bending moment and deflection angle increment. Meanwhile, the stress of the material will change due to strain strengthening effect and strain rate effect, which will lead to the real-time change of the bending moment which resists the deformation. Therefore, bending moment can be regarded as a function of deflection angle $M(\theta)$. The energy consumed by crack propagation is the sum of the energy consumed by each area unit on the fracture section. The energy consumed on the area unit can be expressed as a function of position $\gamma(x, y)$. To sum up, each item at the right side of equation (1) can be expressed as

$$
\begin{aligned}
& E_{e}=2 \int_{0}^{\theta_{s}} M(\theta) \mathrm{d} \theta, \\
& E_{p}=2 \int_{\theta_{s}}^{\theta_{r}} M(\theta) \mathrm{d} \theta, \\
& E_{c}=\iint_{S} \gamma(x, y) \mathrm{d} x \mathrm{~d} y .
\end{aligned}
$$

Among them, $M(\theta)$ is the real-time bending moment of the specimen during bending deformation; $\gamma(x, y)$ is the energy consumed on the area units at different locations of the cross-section when the crack grows; $\theta_{s}$ is the rotation angle of the specimen at the end of the elastic deformation; $\theta_{f}$ is the angle of the specimen when crack initiated at the backside of the specimen; $S$ is the area of the crack propagation section.

Thus, equation (1) can be expressed as

$$
A=2 \int_{0}^{\theta_{s}} M(\theta) \mathrm{d} \theta+2 \int_{\theta_{s}}^{\theta_{r}} M(\theta) \mathrm{d} \theta+\iint_{S} \gamma(x, y) \mathrm{d} x \mathrm{~d} y .
$$

In the elastic deformation stage, for the simply supported rectangular cross-section structure, the bending moment of the cross-section is 


$$
M(\theta)=\sigma_{\max }(\theta) \frac{w h^{2}}{6} .
$$

Among them, $\sigma_{\max }(\theta)$ is the maximum stress on the section, in the elastic stage; its size is related to the bending deformation; $w$ is the width of the cross-section of the notch center; $h$ is the thickness of the cross-section of the notch center.

Substituting equation (6) to equation (2) gives

$$
E_{e}=\frac{w h^{2}}{3} \int_{0}^{\theta_{s}} \sigma_{\max }(\theta) \mathrm{d} \theta
$$

In the elastic stage, the maximum stress and strain on the notch cross-section increase linearly with the deflection angle until the elastic limit is reached, so it can be obtained from equation (7) that

$$
E_{e}=\frac{w h^{2} \sigma_{s} \theta_{s}}{6} .
$$

Among them, $\sigma_{s}$ is the yield strength of the material.

According to mechanics of materials, the deflection angle at the end of elastic deformation stage is

$$
\theta_{s}=\frac{\sigma_{s} L}{4 h E}
$$

Among them, $L$ is the span between the support ends of the simply supported structure. $E$ is young's modulus of the material.

Substituting equation (9) into equation (8), the energy dissipation in the elastic stage is

$$
\begin{aligned}
E_{e} & =2 \int_{0}^{\theta_{s}} M(\theta) \mathrm{d} \theta \\
& =\frac{w h \sigma_{s} \varepsilon_{s} L}{24}
\end{aligned}
$$

where $\varepsilon_{s}$ is the strain when the material reaches the elastic limit.

Regarding $\alpha_{\mathrm{e}}$ as an elastic index reflects the ability of material to absorb energy in elastic stage,

$$
\alpha_{e}=\frac{\sigma_{s} \varepsilon_{s}}{24} .
$$

Substituting equation (11) into equation (10) gives

$$
E_{e}=\alpha_{e} w h L
$$

When the maximum stress on the cross-section reaches the yield strength of the material, the elastic deformation generally turns into plastic deformation. After entering the plastic deformation stage, considering strain strengthening effects and strain rate effect, mechanical properties of the material show a high degree of nonlinear characteristics during the dynamic deformation process [10]. Therefore, the real bending moment at the bending section changes in real time, behaving as a function of deflection angle $\theta$. The realtime bending moment of cross-section in plastic deformation stage can be written as a product of correction coefficient $k_{\mathrm{r}}(\theta)$ and the ultimate plastic moment of crosssection $M_{\mathrm{p}}$, that is,

$$
M(\theta)=k_{r}(\theta) M_{p}
$$

The ultimate plastic bending moment $M_{p}$ is calculated by the static yield strength of the material and the geometric size of the notched section, reflecting the ultimate bending moment when the material on the section theoretically yields completely under static bending. $M_{p}$ does not change in the deformation process. The actual change of bending moment during deformation is considered by multiplying the correction coefficient function $k_{\mathrm{r}}(\theta)$.

Substituting equation (13) to equation (3), we can get

$$
E_{p}=2 M_{p} \int_{\theta_{s}}^{\theta_{r}} k_{r}(\theta) \mathrm{d} \theta .
$$

It is difficult to obtain the analytical form of the strain strengthening effect and strain rate effect of the material in the process of plastic deformation, but the integral result can be replaced by the average value multiplied by the total deformation, that is,

$$
\int_{\theta_{s}}^{\theta_{r}} k_{r}(\theta) \mathrm{d} \theta=k_{\mathrm{ave}} \Delta \theta .
$$

Among them, $k_{\text {ave }}$ is the mean value in the process of change $k_{r}(\theta)$.

Substituting equation (15) to equation (14), it gives

$$
E_{p}=2 k_{\mathrm{ave}} M_{p} \Delta \theta
$$

For a rectangular cross-section structure, the ultimate moment of the section is

$$
M_{p}=\frac{\sigma_{s} w h^{2}}{4} .
$$

Different from elastic deformation, the plastic deformation of the specimen after impact is limited to a local position. The plastic area of a rectangular cross-section specimen is assumed to be two symmetrically distributed triangles, as shown in Figure 2.

When plastic deformation occurs, the relation between deflection angle increment $\Delta \theta$ and curvature $k$ is

$$
\kappa \approx \frac{2 \Delta \theta}{l_{h}},
$$

where $l_{h}$ is the largest length of plastic area, as shown in Figure 2.

It should be noted that the local length of plastic area $l_{h}$ shall change during the deformation. However, for mathematical simplicity, some fixed values of $l$ are recommended. In the case of rectangular cross-section beam, $l_{\mathrm{h}}$ roughly equals to the thickness of bending section [11,12].

The relation between the maximum strain in the bending section and the curvature is 


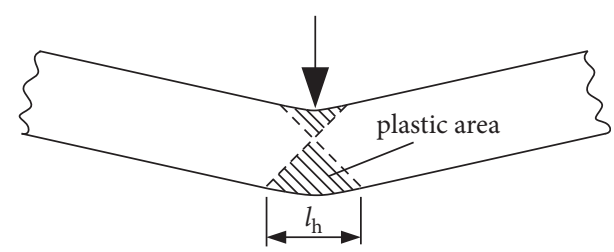

Figure 2: Plastic area at the impact point of a rectangular crosssection specimen.

$$
\varepsilon_{\max }=\kappa z_{\max }=\kappa \frac{h}{2}
$$

where $z_{\max }$ is the distance from the notch to the neutral surface.

If $\varepsilon_{\max }$ increases to fracture strain $\varepsilon_{\mathrm{f}}$, crack initiation occurs, and fracture strain $\varepsilon_{\mathrm{f}}$ is a mechanical property of material itself. Let $\varepsilon_{\max }=\varepsilon_{f}$; from equation (18) and (19), the deflection angle increment when crack initiation occurs can be derived

$$
\Delta \theta=f\left(\varepsilon_{f}\right)=\varepsilon_{f} \frac{l_{h}}{h}
$$

From equations (16) (20), the energy consumed by plastic deformation can be derived

$$
E_{p}=2 \int_{\theta_{s}}^{\theta_{r}} M(\theta) \mathrm{d} \theta=k_{\mathrm{ave}} \sigma_{s} \varepsilon_{f} \frac{w h l_{h}}{2} .
$$

Let

$$
\alpha_{t}=k_{\mathrm{ave}} \sigma_{s} \varepsilon_{f} .
$$

So, equation (21) can be written as

$$
E_{p}=\alpha_{t} \frac{w h l_{h}}{2}
$$

Among them, $\alpha_{t}$ is the intrinsic impact toughness, which is defined by equation (22) to reflect the toughness of material itself in the dynamic process.

Similarly, the average value is used to simplify the expression of energy consumed during crack propagation, which can be written as the product of average energy per unit area and fracture cross-sectional area. Therefore, equation (4) can be simplified as follows:

$$
E_{c}=2 \iint_{S} \gamma(x, y) \mathrm{d} x \mathrm{~d} y=2 \gamma_{\text {ave }} w h .
$$

Among them, $\gamma_{\text {ave }}$ is the average energy consumption per unit area during crack propagation, reflecting the ability of the material to resist crack propagation.

Substituting equations (12), (23), and (24) to equation (5), the simplified mathematical model of the impact work for rectangular cross-section structure can be obtained

$$
A=\alpha_{e} w h L+\alpha_{t} \frac{w h l_{h}}{2}+2 \gamma_{\mathrm{ave}} w h
$$

Taking the local length of plastic deformation $l_{h} \approx h$ $[11,12]$, we substitute it to equation (25) and derive

$$
A \approx \alpha_{t} \frac{w h^{2}}{2}+\left(2 \gamma_{\mathrm{ave}}+\alpha_{e} L\right) w h
$$

\section{Experimental Verification of Impact Work Mathematical Model}

The mathematical model described by equation (26) was verified by the impact experiments of 921A and Q235B steel.

3.1. Impact Test Equipment and Test Materials. In order to verify the validity of equation (26), a series of impact tests on rectangular cross-section specimens with different sizes and regression analyses of the impact test results were carried out.

The impact test was conducted at room temperature, and JB-300B pendulum impact tester was selected to carry out impact loading on the specimen. The geometry of the specimens is shown as Figure 3. The span between supports $L$ was $40 \mathrm{~mm}$; the length of all specimens was $55 \mathrm{~mm}$. To control the crack position, a V-notch, whose angle is $45^{\circ}$ and depth is $2 \mathrm{~mm}$, was made at the center of each specimen. The radius of curvature at the bottom of the notch is $0.25 \mathrm{~mm}$. Ligament length $h$ is the width of specimen which really takes part in the baring. All specimens were processed by wire cutting and polished to make the roughness meet ASTM A370 standard requirements. Oil pollution was removed with acetone.

Ultra-high strength steel $921 \mathrm{~A}$ is chosen for impact test in this study; its basic mechanical properties are measured as shown in Table 1.

Three impact tests were conducted on each impact specimen of the same size and material, and the average of the three tests was taken as the final result.

3.2. Analysis of Impact Test Results. The impact test results of specimens made of 921A steel are shown in Table 2.

According to the physical meaning of each parameter in equation (26), boundary conditions of parameters were given in Table 3 for the regression analysis. The intrinsic impact toughness $\alpha_{\mathrm{t}}$ and average energy consumption per unit area $\gamma_{\text {ave }}$ must be positive. The value of $\alpha_{\mathrm{e}}$ can be calculated by substituting the yield strength and yield strain of each material in equation (11).

Nonlinear double independent variable regression analyses were conducted with software Mathematica, and the analysis results are shown in Table 4 . The R-square value is close to 1 , indicating the effectiveness of fitting. However, the value of $\gamma_{\text {ave }}$ is close to 0 , and the $P$ value is close to 1 , showing that the value of $\gamma_{\text {ave }}$ is extremely insignificant. Compared with the energy dissipating during elastic deformation and the plastic deformation energy, the surface 


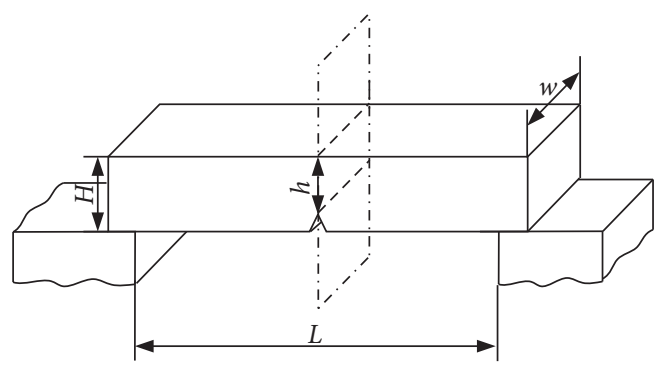

FIGURE 3: Dimensions of impact specimen.

TABle 1: Basic mechanic properties of tested materials.

\begin{tabular}{lccc}
\hline Material $(\mathrm{A})$ & $\sigma_{\mathrm{s}}(\mathrm{MPa})$ & $\sigma_{\mathrm{b}}(\mathrm{MPa})$ & $e(\%)$ \\
\hline 921 & 665 & 729 & 25.5 \\
\hline
\end{tabular}

TABLe 2: Impact test results of 921A steel.

\begin{tabular}{lccc}
\hline Specimen number & $w(\mathrm{~mm})$ & $h(\mathrm{~mm})$ & $A(\mathrm{~J})$ \\
\hline A1 & 10 & 3 & 30 \\
A2 & 10 & 4 & 46 \\
A3 & 10 & 6 & 102 \\
A4 & 10 & 6 & 108 \\
A5 & 10 & 6.5 & 110 \\
A6 & 10 & 7 & 130 \\
A7 & 10 & 8 & 202 \\
A8 & 10 & 8 & 210 \\
A9 & 10 & 9 & \\
A10 & 6 & 8 & \\
A11 & 6.5 & 8 & \\
A12 & 8 & 8 & \\
A13 & 9.5 & 8 & \\
A14 & 10 & 8 & \\
A15 & 12 & 8 & \\
\hline
\end{tabular}

TABLE 3: Boundary conditions constrained by physical significance.

\begin{tabular}{lcc}
\hline$\alpha_{\mathrm{t}}\left(\mathrm{mJ} / \mathrm{mm}^{3}\right)$ & $\alpha_{\mathrm{e}}\left(\mathrm{mJ} / \mathrm{mm}^{3}\right)$ & $\gamma_{\text {ave }}\left(\mathrm{mJ} / \mathrm{mm}^{2}\right)$ \\
\hline$>0$ & 0.0554 & $>0$ \\
\hline
\end{tabular}

TABLE 4: Regression analysis results.

\begin{tabular}{lccc}
\hline & Fitting result & $P$ value & $R$-squared \\
\hline$\alpha_{\mathrm{t}}$ & 579.765 & 0.0002 & - \\
$\alpha_{\mathrm{e}}$ & 0.0554 & 9.99482 & 0.989375 \\
$\gamma_{\mathrm{ave}}$ & 0.00034 & \\
\hline
\end{tabular}

energy of materials used in this research is much smaller than the first two with a couple of orders of magnitude differences [13]. Therefore, as a result of regression analysis, the insignificance of $\gamma_{\text {ave }}$ is reasonable, suggesting that $\gamma_{\text {ave }}$ may be ignored under low accuracy requirement.

Substituting sizes of the specimens and the fitting results of $\alpha_{\mathrm{e}}$ and $\alpha_{\mathrm{t}}$ to equations (12) and (23), respectively, we found out that for materials with appreciable plasticity, the energy absorbed by plastic deformation is much larger than that of elastic deformation, which was commonly accepted by existing researches [14].
Substituting the fitting results of $921 \mathrm{~A}$ steel into equation (26), we obtained the relationship between $a$ and $H$ (Figure 4(a)) and the relationship between $a$ and $w$ (Figure 4(b)). Meanwhile, impact test results were also marked in the corresponding coordinates for comparation, as shown in Figure 4.

It can be seen from Figure 4 that the results of impact test were all distributed near the curve base on equation (26). The average difference between the results evaluated with equation (26) and those obtained by impact tests is $10.54 \%$. Considering the considerable discreteness of impact test itself, the 


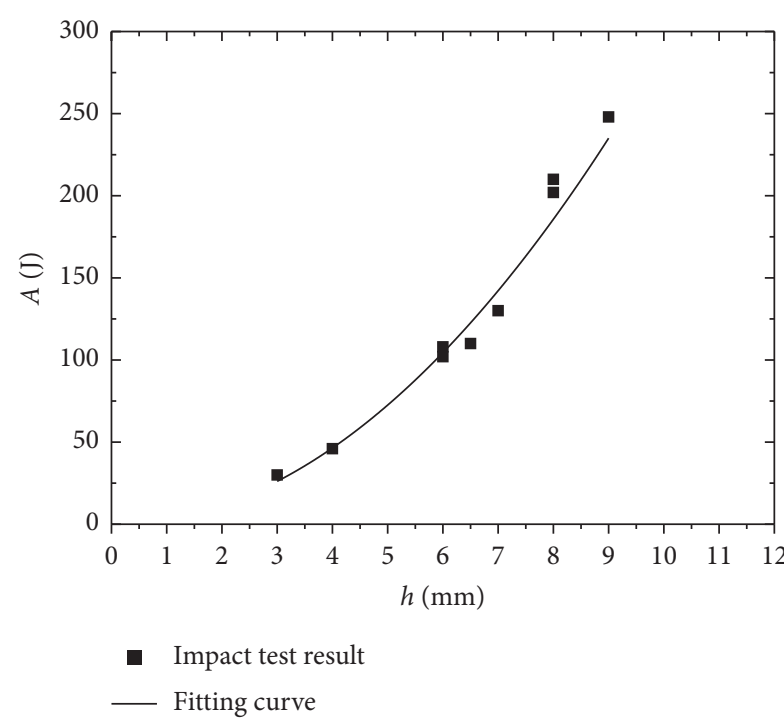

(a)

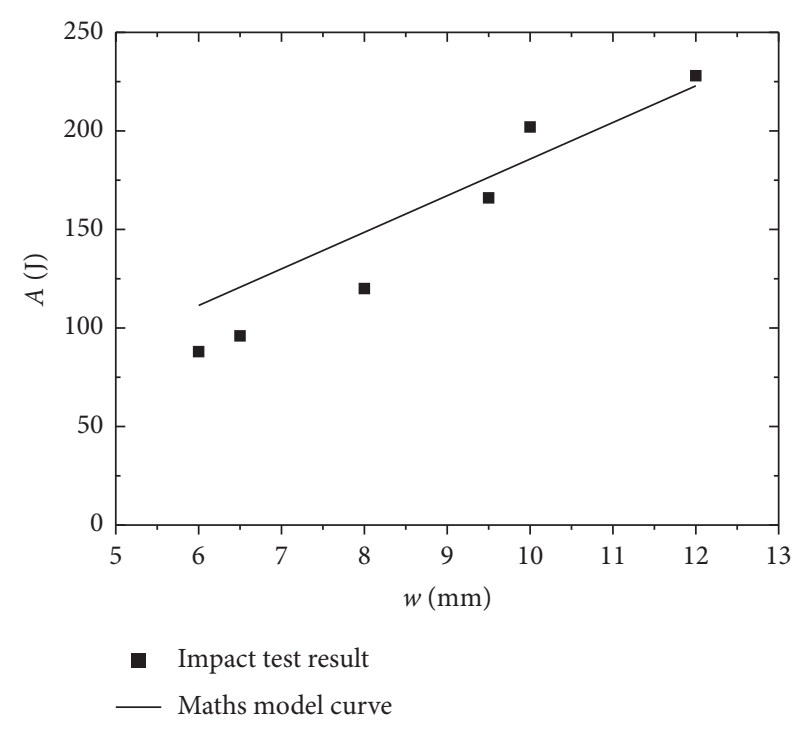

(b)

Figure 4: Comparison of evaluations based on model and impact test results for 921 steel. (a) A1 A9 specimen impact test results. (b) A10 A15 specimen impact test results.

accuracy and validity of the mathematical model described by equation (26) can be acceptable. Furthermore, the relation between ligament length $h$ and impact energy $A$ was quadratic approximately and the relationship between thickness $\mathrm{W}$ and impact energy $a$ is approximately linear, which is in good agreement with the mathematical model [15].

\section{Discussion}

The mathematical model of impact energy illuminated the relation between mechanical properties of the material itself, the geometric dimensions of the structure, and the impact work. Thus, it is possible to estimate the impact energy of different size structures with known mechanical properties parameters of the materials. If the impact energy of a certain structure is taken as an index to evaluated impact bearing capability of the structure, this model can be used as a design basis or a tool of reliability evaluation [16].

Furthermore, the commonly used parameters $A_{\mathrm{k}}$ and $\alpha_{\mathrm{k}}$ are influenced by the geometry of specimen. Thus, the comparison of $A_{\mathrm{k}}$ or $\alpha_{\mathrm{k}}$ between specimens of different or nonstandard sizes cannot reflect their difference in toughness [17]. The intrinsic impact toughness $\alpha_{\mathrm{t}}$ in this paper has no relation with the geometry of specimen, which can be regarded as a mechanical property of material itself. Furthermore, $\alpha_{\mathrm{t}}$ can be obtained by regression analysis based on the mathematical model constructed in this paper [18]. Considering the convenience of impact test, $\alpha_{\mathrm{t}}$ has the potential to be applied to evaluate the toughness of materials such as $K_{\text {Ic }}$ and $K_{\text {Id. }}$.

It is worth noting that the mathematical model of impact energy in this paper is established for materials with obvious plasticity. For materials with little plasticity, the mathematical model proposed in this study is not applicable [18]. The mathematical model proposed in this study did not consider the inertia effect of the structure under higher rate impact which needs to be further studied.

\section{Conclusion}

(1) By analyzing the dynamic response process of the V-shaped gap reduced by the transverse impact of the placed hammer, a mathematical model can be established to estimate the impact energy of different size fragments. This study reveals that the impact energy is linearly related to the width of the notch cross-section. The rules related to the second-time nonlinearity of the thickness have been verified by a series of impact defects.

(2) The inherent impact toughness is independent of the size and geometry of the material, which can be called the inherent impact toughness of the material itself. When comparing the volumes of different materials, it is no longer necessary to use specimens of the same standard size for impact test.

(3) When the structure is used below the ductile-brittle transition temperature and high-speed impact load, the mathematical model established in this study cannot be used in theory, which needs to be considered in the structural inertia.

\section{Data Availability}

The labeled dataset used to support the findings of this study are available from the corresponding author upon request.

\section{Conflicts of Interest}

The authors declare no conflicts of interest. 


\section{Acknowledgments}

This work was supported by Youth Program of National Natural Science Foundation of China (51605116).

\section{References}

[1] K. K. Mathur, A. Needleman, and V. Tvergaard, "3D analysis of failure modes in the Charpy impact test," Modelling and Simulation in Materials Science and Engineering, vol. 2, no. 3A, pp. 617-635, 1994.

[2] Y. Nakamura, M. Yamaguchi, and M. Okubo, "Instrumented charpy impact test of epoxy resin filled with irregular-shaped silica particles," Polymer Engineering \& ence, vol. 33, no. 5, pp. 279-284, 2010.

[3] H. Kurishita, H. Kayano, M. Narui, M. Yamazaki, Y. Kano, and I. Shibahara, "Effects of V-Notch dimensions on Charpy impact test results for differently sized miniature specimens of ferritic steel," Materials Transactions, JIM, vol. 34, no. 11, pp. 1042-1052, 1993.

[4] Y. Takashima, M. Ohata, and F. Minami, "Evaluation method for fracture mechanics-based material toughness from Charpy impact test," Materials Science Forum, vol. 512, pp. 61-66, 2006.

[5] C. H. Lee, H. S. Shin, K. T. Park, and S. H. Yang, "Evaluation of the applicability of structural steels to cold regions by the Charpy impact test," Journal of Korean Society of Steel Construction, vol. 23, no. 4, pp. 483-491, 2011.

[6] K. Toshiro, Y. Isamu, and N. Mitsuo, "Evaluation of dynamic fracture toughness parameters by instrumented Charpy impact test," Engineering Fracture Mechanics, vol. 24, no. 5, pp. 773-782, 1986.

[7] S. H. Kim, Y. W. Park, S. S. Kang, and H. D. Chung, "Estimation of fracture toughness transition curves of RPV steels from Charpy impact test data," Nuclear Engineering and Design, vol. 212, no. 1-3, pp. 49-57, 2002.

[8] R. Chaouadi and A. Fabry, "On the utilization of the instrumented Charpy impact test for characterizing the flow and fracture behavior of reactor pressure vessel steels," From Charpy To Present Impact Testing, vol. 30, pp. 103-117, 2002.

[9] P. R. Sreenivasan and S. L. Mannan, "Plastic n-factor for threepoint bend specimens: analysis of instrumented Charpy impact test results for AISI 308 weld and AISI 316 stainless steels," International Journal of Fracture, vol. 101, no. 3, pp. 215-228, 2000.

[10] N. S. Cannon and D. S. Gelles, "Charpy impact test results from low activation alloys irradiated to $10 \mathrm{dpa}$ at $365^{\circ} \mathrm{C}$," Journal of Nuclear Materials, vol. 186, no. 1, pp. 68-76, 1991.

[11] H. Kikuchi, M. Harada, K. Ara, Y. Kamada, S. Kobayashi, and S. Takahashi, "Development of apparatus for magnetic measurements of Charpy impact test pieces," Journal of Materials Processing Technology, vol. 181, no. 1-3, pp. 190-193, 2007.

[12] M. M. Shokrieh, M. A. Torabizadeh, and A. Fereidoon, "Dynamic failure behavior of glass/epoxy composites under low temperature using Charpy impact test method," Indian Journal of Engineering \& Materials ences, vol. 18, no. 3, pp. 211-220, 2011.

[13] O. S. Lee and S. K. Hong, "Dynamic fracture characteristics of highly brittle materials by using instrumented Charpy impact test," KSME International Journal, vol. 11, no. 5, pp. 513-520, 1997.

[14] T. Iwadate and H. Takemata, "Prediction method of fracture toughness KIC transition curve from Charpy V-Notch impact test results," Journal of the Society of Materials Science, Japan, vol. 41, no. 467, pp. 1241-1247, 1992.

[15] G. V. Kozlov, R. A. Shetov, and A. K. Mikitayev, "Determination of the limit of forced elasticity in the Charpy impact test," Polymer Science U.S.S.R.vol. 29, no. 9, pp. 2205-2207, 1987.

[16] K. Seo, F. Nogata, and J.-i. Masaki, "The absorbed energy in Charpy impact test for specimens with mechanical heterogeneity," Journal of the Society of Materials Science, Japan, vol. 35, no. 399, pp. 1405-1410, 1986.

[17] J. A. Wang and N. Rao, "New methodologies for developing radiation embrittlement models and trend curves of the Charpy impact test data," Journal of ASTM International, vol. 1, no. 9, p. 19, 2004.

[18] T. Otsuka, K. Hashizume, and M. Sugisaki, "Charpy impact test of oxidized and hydrogenated zircaloy using a thin strip specimen," Journal of Nuclear Science and Technology, vol. 41, no. 3, pp. 247-251, 2004. 